\title{
Controlled phase gate for solid-state charge-qubit architectures
}

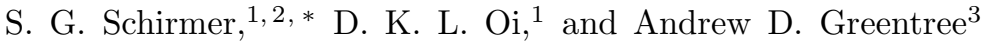 \\ ${ }^{1}$ Department of Applied Mathematics and Theoretical Physics, \\ University of Cambridge, Wilberforce Road, Cambridge CB3 OWA, UK \\ ${ }^{2}$ Department of Engineering, University of Cambridge, Trumpington Street, Cambridge, CB2 1PZ, UK \\ ${ }^{3}$ Centre for Quantum Computer Technology, School of Physics, \\ University of Melbourne, Melbourne, Victoria 3010, Australia
}

(Dated: February 1, 2008)

\begin{abstract}
We describe a mechanism for realizing a controlled phase gate for solid-state charge qubits. By augmenting the positionally defined qubit with an auxiliary state, and changing the charge distribution in the three-dot system, we are able to effectively switch the Coulombic interaction, effecting an entangling gate. We consider two architectures, and numerically investigate their robustness to gate noise.

PACS numbers: 03.67.Lx,03.65.Vf,85.35.Be
\end{abstract}

\section{INTRODUCTION}

The search for a workable quantum information processor is an effort that has captivated the attention of researchers in many disciplines. A quantum computer requires individual quantum logic elements, usually qubits, and entangling interactions between these elements 1, 2].

Solid-state proposals are widely seen as being some of the most attractive from the point of view of constructional scalability, i.e., the ability to replicate many qubits. Furthermore, schemes compatible with present semiconductor technologies $[3,4,5,6]$ are especially attractive because of their potential to leverage the associated industrial experience [7, 8].

In this paper we concentrate on charge-based architectures. Such systems were amongst the first proposed for quantum computing 9] and numerous versions have evolved recently 6, 10, 11, 12. We are attracted to charge-based systems for three reasons: (1) proven high-fidelity readout compatible with single-shot operations 13]; (2) potential for high-speed ( picosecond) operations [6]; and (3) the ability to define variable dimensionality Hilbert spaces by appropriate partitioning [14]. The usual mechanism for coupling charge qubits is via the Coulomb interaction. In general, this interaction cannot be controlled without a variation in the charge distribution in the qubits. In this paper we specifically address this issue and show how to make a scalable controlled phase gate that makes use of the Coulomb interaction.

The Coulomb interaction is insensitive to minor variations in the distance between the quantum dots and its strength lends itself to high-speed entangling operations; conversely, its long-range nature makes it difficult to effectively modulate interactions between qubits. Most charge qubit schemes so far proposed implicitly rely on a fixed, always-on Coulomb interaction between qubits 6, 10, 15] Such schemes would necessarily require

*Electronic address: sgs29@cam.ac.uk global control techniques [16, 17, 18], which may be problematic given the strength of the Coulomb interaction.

In the following we first discuss local qubit operations (Sec. III) and introduce the two-qubit interaction Hamiltonian (Sec. III). Then we describe how to realize a controlled phase gate and analyze its operation in terms of dynamic and geometric phases (Sec. IV). Finally, we discuss various practical issues such as the detrimental effects of finite rise and decay times on the gate fidelity and how to correct them (Sec. (V), gate implementation in the presence of practical constraints on pulse lengths and tunnelling rates (Sec. VI), and the effect of noisy controls (Sec.VII) and imperfect architectures (Sec. VIII) on the gate performance.

\section{SINGLE QUBIT SYSTEM}

As will be shown below, a three-dot system is required to modulate the Coulomb interaction. We therefore supplement the canonical charge qubit with an auxiliary state and define a quantum element with three quantum dots and one charge, where the position of the charge on two sites defines the qubit and the third site defines an auxiliary state used for two-qubit interactions. We work only with the ground state of each dot. We further assume that the ground states are energetically close and sufficiently separated from higher-lying excited states, so that the excitation of these states can be neglected. The system can thus be approximated as a three level system with Hamiltonian

$$
\hat{H}=\sum_{d=1}^{3} \epsilon_{d}|d\rangle\langle d|+\hbar \sum_{d^{\prime} \neq d} \mu_{d d^{\prime}} \hat{X}_{d d^{\prime}},
$$

where $|d\rangle$ denotes the ground state of the electron localized in $\operatorname{dot} d$ for $d=1,2,3, \epsilon_{d}$ the energy of state $|d\rangle, \mu_{d d^{\prime}}$ the tunnelling rate between $\operatorname{dots} d$ and $d^{\prime}$ (for $d^{\prime} \neq d$ ), and $\hat{X}_{d d^{\prime}}=|d\rangle\left\langle d^{\prime}|+| d^{\prime}\right\rangle\langle d|$. We shall assume that we can control the ground state energies $\epsilon_{d}$ and the tunnelling 
electric potential \& ground state energy levels

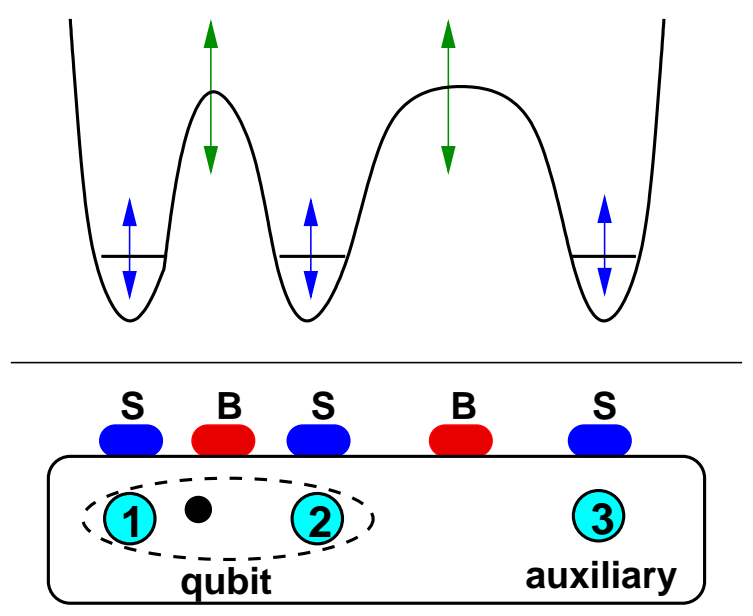

FIG. 1: Schematic of a single qubit-plus-auxiliary unit (bottom) and electronic potential (top). The large circles represent the quantum dots, the black dot the shared electron. $S$ and $B$ surface electrodes serve to shift the ground state energy of the dots and change the height of the tunnelling barriers and thus the tunnelling rates between them.

rates $\mu_{d d^{\prime}}$, e.g., by varying the voltages applied to several control electrodes as illustrated in Fig. [1]

To implement local unitary operations, we inhibit tunnelling to the auxiliary site by raising the barrier between dots 2 and 3 (and/or 1 and 3 if applicable), or increasing the ground state energy of the auxiliary dot 3. In practice, the precise functional dependence of $\epsilon_{d}$ and $\mu_{d d^{\prime}}$ on the control voltages applied should be determined experimentally using Hamiltonian identification techniques [19]. When $\mu_{13}=\mu_{23}=0$ the local Hamiltonian can be rewritten as

$$
\hat{H}=\epsilon_{1}|1\rangle\left\langle 1\left|+\epsilon_{2}\right| 2\right\rangle\left\langle 2\left|+\hbar \mu_{12} \hat{X}_{12}+\epsilon_{3}\right| 3\right\rangle\langle 3|,
$$

and we can realize arbitrary unitary operations on the qubit subspace by changing the energy levels $\epsilon_{d}$ and the tunnelling rate $\mu_{12}$. For instance, shifting the energy level of dot 2 by $\epsilon_{2}(t)$ for $t_{0} \leq t \leq t_{1}$ results in a local phase rotation

$$
\hat{U}_{2}(\phi)=|1\rangle\left\langle 1\left|+e^{-i \phi}\right| 2\right\rangle\langle 2|+| 3\rangle\langle 3|
$$

with $\phi=\int_{t_{0}}^{t_{1}} \epsilon_{2}(t) / \hbar d t$. Effecting a tunnelling rate of $\mu_{12}$ for $t_{0} \leq t \leq t_{1}$ gives

$$
\hat{U}_{12}(\alpha)=\cos (\alpha) \hat{I}_{12}-i \sin (\alpha) \hat{X}_{12}+|3\rangle\langle 3|,
$$

where the rotation angle is $\alpha=\int_{t_{0}}^{t_{1}} \mu_{12}^{(k)}(t) d t$ and $\hat{I}_{12}=$ $|1\rangle\langle 1|+| 2\rangle\langle 2|$. By combining two phase rotations on the 2nd dot with a tunnelling gate between sites 1 and 2, for example, we can implement any local unitary operation on the qubit subspace modulo global phases [2]

$$
\hat{U}\left(\phi_{1}, \alpha, \phi_{2}\right)=\hat{U}_{2}\left(\phi_{2}\right) \hat{U}_{12}(\alpha) \hat{U}_{2}\left(\phi_{1}\right)
$$

$$
=\left(\begin{array}{rr|r}
\cos (\alpha) & -i e^{-i \phi_{1}} \sin (\alpha) & 0 \\
-i e^{-i \phi_{2}} \sin (\alpha) & e^{-i\left(\phi_{1}+\phi_{2}\right)} \cos (\alpha) & 0 \\
\hline 0 & 0 & 1
\end{array}\right),
$$

For example, a Hadamard transform on the qubit subspace corresponds to

$$
\hat{H}=\frac{1}{\sqrt{2}}\left(\begin{array}{rr}
1 & 1 \\
1 & -1
\end{array}\right)=\hat{U}\left(\phi_{2}=-\frac{\pi}{2}, \alpha=\frac{\pi}{4}, \phi_{1}=-\frac{\pi}{2}\right) .
$$

It is possible to optimize the implementation of some local unitary operations by simultaneously changing multiple control parameters. See appendix A

\section{INTERACTION HAMILTONIAN}

To achieve entangling operations it is necessary to change the charge distribution of adjacent elements. Although almost any interaction will lead to entanglement [20], the resulting dynamics may be neither easy to utilize, nor robust against noise.

Rather than choose an arbitrary interaction, it is more useful to consider geometries where the action of the Coulomb force on the qubit states is trivial. One way this might be achieved is by using shielding to eliminate direct interactions between qubits but not between the auxiliary sites as shown in Fig.2(a). Such a system could be fabricated in a GaAs 2DEG system [11, 21] or a pillar system [22], for instance. An alternative is to choose a geometry for which the effect of the Coulomb interaction on the dynamics of the qubit subspace is trivial, resulting only in a global phase factor, as in Fig. 2 (b). To fabricate such a structure, we would propose an extension of the atomic placement techniques used for Si:P systems [23, 24]. Both designs have the advantage of allowing the implementation of controlled two-qubit gates using simple pulse sequences. Note that using auxiliary sites has also been proposed as a way to allow generalized readout of quantum information in different bases [25].

The Hilbert space for two qubit-plus-auxiliary units is spanned by the states $\left|d d^{\prime}\right\rangle$ for $d, d^{\prime}=1,2,3$, where $|d\rangle_{k}$ denotes the $d$ th basis state for the $k$ th unit, and $\left|d d^{\prime}\right\rangle=|d\rangle_{1} \otimes\left|d^{\prime}\right\rangle_{2}$ are the tensor product states as usual. The total Hamiltonian of this system is

$$
\hat{H}=\hat{H}^{(1)} \otimes \hat{I}_{3}+\hat{I}_{3} \otimes \hat{H}^{(2)}+\hat{H}_{C}
$$

where $\hat{I}_{3}$ is the identity matrix in dimension three, $\hat{H}^{(k)}$ for $k=1,2$ is the local Hamiltonian for the $k$ th qubitplus-auxiliary unit specified in Eq. (1), and $\hat{H}_{C}$ is the Coulomb Hamiltonian

$$
\hat{H}_{C}=\sum_{d, d^{\prime}=1}^{3} \gamma_{d d^{\prime}}\left|d d^{\prime}\right\rangle\left\langle d d^{\prime}\right|
$$



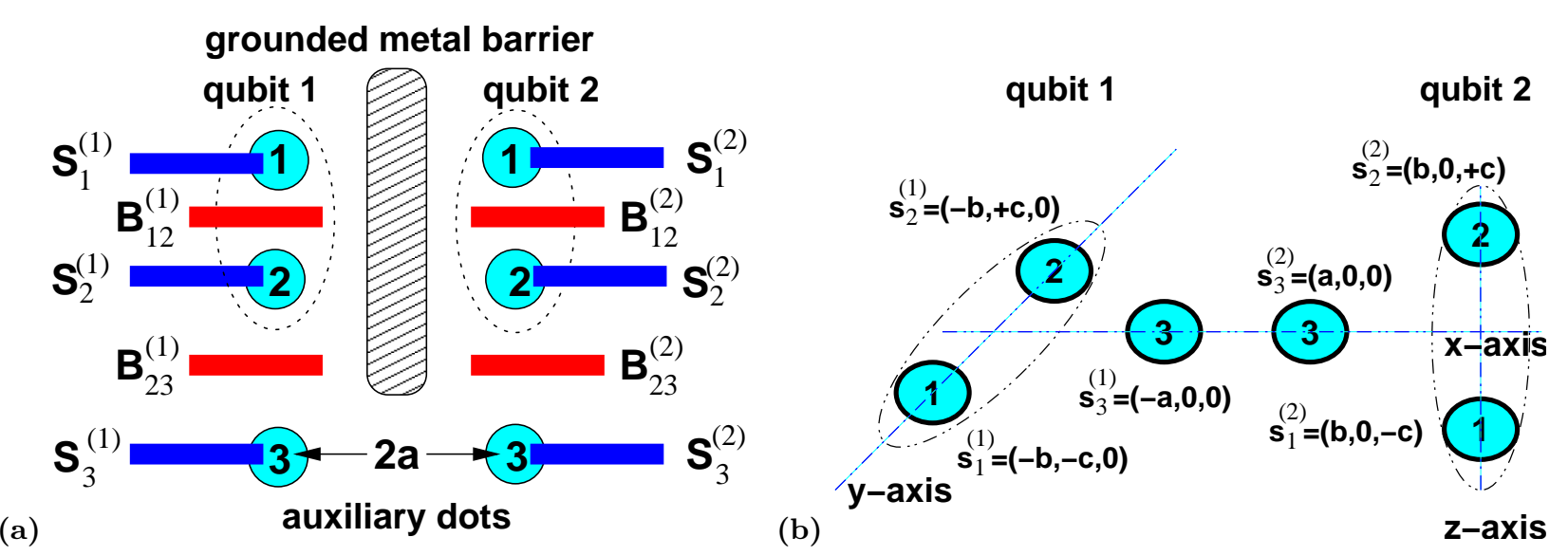

FIG. 2: (a) Two-qubit system with two auxiliary sites comprised of six quantum dots (filled circles). A grounded metal barrier shields the Coulomb interaction between nearby quantum dots except between the two auxiliary sites (3). The thick (red and blue) lines indicate the surface control electrodes $B_{d d^{\prime}}^{(k)}$ and $S_{d}^{(k)}$, respectively. (b) 3D geometry for a two-qubit system with auxiliary sites, for which the Coulomb interaction between the two charges is constant if both are confined to their respective qubit subspace comprised of dots 1 and 2, but differs from the Coulomb coupling between the auxiliary dots provided that $\sqrt{4 b^{2}+2 c^{2}} \neq 2 a$. Note that the inter-dot distances satisfy $\left\|\mathbf{s}_{d}^{(1)}-\mathbf{s}_{d^{\prime}}^{(2)}\right\|=\sqrt{4 b^{2}+2 c^{2}}$ and $\left\|\mathbf{s}_{3}^{(1)}-\mathbf{s}_{d}^{(2)}\right\|=\left\|\mathbf{s}_{d}^{(1)}-\mathbf{s}_{3}^{(2)}\right\|=$ $\sqrt{(a+b)^{2}+c^{2}}$ for $d, d^{\prime}=1,2$.

The Coulomb interaction energies $\gamma_{d d^{\prime}}$ are given by

$$
\gamma_{d d^{\prime}}=\frac{e^{2}}{4 \pi \epsilon}\left\|\mathbf{s}_{d}^{(1)}-\mathbf{s}_{d^{\prime}}^{(2)}\right\|^{-1},
$$

where $\mathbf{s}_{d}^{(k)}$ denotes the position of the $d$ th quantum dot in the $k$ th qubit-plus-auxiliary unit, $\epsilon$ is the applicable dielectric constant, and $e$ is the electron charge. In pure silicon we have $\epsilon=11.8 \epsilon_{0}$, where $\epsilon_{0}$ is the dielectric constant in vacuum.

If two sites $\mathbf{s}_{d}^{(1)}$ and $\mathbf{s}_{d^{\prime}}^{(2)}$ are separated by a sufficiently thick, grounded metal barrier then $\gamma_{d d^{\prime}}=0$. Hence, the Coulomb interactions for the shielded 2D geometry in Fig. 2 (a) effectively vanish except for $\gamma_{33}$. Similarly, for the 3D geometry shown in Fig. 2 (b), symmetry implies

$$
\begin{aligned}
& \gamma_{11}=\gamma_{12}=\gamma_{21}=\gamma_{22} \equiv \gamma_{1}, \\
& \gamma_{13}=\gamma_{23}=\gamma_{31}=\gamma_{32} \equiv \gamma_{2} .
\end{aligned}
$$

We can cancel the effect of the Coulomb interaction between qubit states by applying suitable bias voltages to the energy shift gates to offset the energy levels $\epsilon_{1}^{(k)}$ and $\epsilon_{2}^{(k)}$ by $-\gamma_{1} / 2$ and $\epsilon_{3}^{(k)}$ by $\gamma_{1} / 2-\gamma_{2}$ for $k=1,2$. Thus, the Coulomb interaction Hamiltonian becomes

$$
\hat{H}_{C}=\gamma_{\text {eff }}|33\rangle\langle 33|,
$$

where $\gamma_{\text {eff }}$ is the effective Coulomb coupling between the auxiliary states, i.e., $\hat{H}_{C}$ acts trivially on the system except if both electrons are in the auxiliary states.

For a $2 \mathrm{D}$ architecture with shielding $\gamma_{\text {eff }}$ will usually be less than the free-space Coulomb interaction $\gamma_{33}$ due to screening and image charges. For a 3D geometry with energy offsets $\gamma_{\text {eff }}$ will be less than or equal to $\gamma_{33}-2 \gamma_{2}+$ $\gamma_{1}$, with equality if the screening due to control electrodes etc. is negligible.
For convenience, we choose the effective Coulomb energy $\gamma_{\text {eff }}$ between the auxiliary sites as the unit of energy such that the free evolution Hamiltonian of the two-qubit plus auxiliary system is $\hat{H}_{0}=|33\rangle\langle 33|$. All tunnelling rates are given in units of $\gamma_{\text {eff }} / \hbar$ and the canonical time unit is $\hbar / \gamma_{\text {eff }}$.

\section{CONTROLLED TWO-QUBIT PHASE GATE}

To motivate the design of a two-qubit phase gate, we note that the phase acquired by an electron tunnelling between two quantum dots depends on the energy difference between them. In our system the energy differences are determined by a combination of energy bias voltages and the Coulombic interaction between the auxiliary sites. Hence, by adjusting the voltage on the energy bias gate $S_{2}^{(1)}$, for instance, we can shift of the energy of state $|2\rangle_{1}$ such that the energy differences between states $|21\rangle$ and $|31\rangle$, and $|23\rangle$ and $|33\rangle$, respectively, are equal in magnitude but opposite in sign, as illustrated in Fig. 3. In this case the phase acquired by a charge tunnelling between dots 2 and 3 of qubit 1 will have the same magnitude regardless of the population of state $|3\rangle_{2}$, but the latter will determine its sign. This observation is central to the operation of our controlled phase gate.

To achieve a maximally entangling gate, the acquired phases in both cases must differ by an integer multiple of $\pi$. Finally, except for the acquired phase, the charge must return to its initial state $|2\rangle_{1}$ at the end of the tunnelling process, i.e., no population must remain in the auxiliary state $|3\rangle_{1}$. Combining all of these requirements leads to 


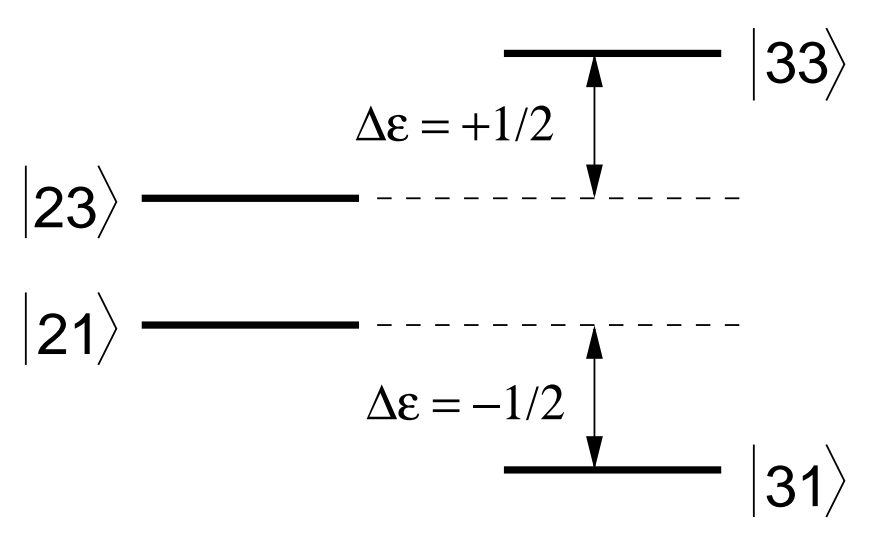

FIG. 3: Energy level configuration during the second step: By applying an energy bias of $\epsilon_{2}^{(1)}=1 / 2$ we ensure that the energy gap between states $|23\rangle$ and $|33\rangle$, and $|21\rangle$ and $|31\rangle$ is $\pm 1 / 2$, respectively.

the following expressions for the tunnelling rate

$$
\mu_{23}^{(1)}=\frac{1}{4} \sqrt{\left(\frac{2 n}{2 k-1}\right)^{2}-1}
$$

for suitable positive integers $n$ and $k$ satisfying $2 n>$ $2 k-1$, and tunnelling time

$$
\tau_{2}=\frac{4 \pi n}{\sqrt{16\left(\mu_{23}^{(1)}\right)^{2}+1}}=2 \pi(2 k-1) .
$$

A detailed explanation of these results is provided in appendices $\mathrm{C}$ and D. Furthermore, the phase acquired by state $|21\rangle$ is $[2 n-(2 k-1)] \pi / 2$, while that of state $|23\rangle$ is $[2 n+(2 k-1)] \pi / 2$, and hence the phase difference is $(2 k-1) \pi \equiv \pi$ as desired.

In the absence of constraints on the tunnelling rates and pulse lengths, the gate operation time is optimized if we choose $n=k=1$ and $\mu_{23}^{(1)}=\sqrt{3} / 4$. For details about how to choose $n$ and $k$ when there are constraints on the tunnelling rates and pulse lengths, see appendix E

More explicitly, a maximally entangling controlled twoqubit phase gate

$$
\begin{aligned}
\hat{U}_{\text {phase }} & =|11\rangle\langle 11|+| 12\rangle\langle 12|+| 21\rangle\langle 21|-| 22\rangle\langle 22| \\
& =\operatorname{diag}\left(\hat{I}_{2}, \hat{Z}_{12}\right) .
\end{aligned}
$$

can be realized as follows:

1. Acting on the second qubit, swap the populations of the states $|2\rangle_{2}$ and $|3\rangle_{2}$ by lowering the tunnelling barrier between the 2nd and 3rd quantum dot for time $\tau_{1}=\pi /\left(2 \mu_{23}^{(2)}\right)$, where $\mu_{23}^{(2)}$ is the tunnelling rate.

2. Acting on the first qubit, simultaneously raise the ground state energy of the 2 nd dot by $\epsilon_{2}^{(1)}=1 / 2$ and lower the tunnelling barrier between the 2nd and 3rd dot to achieve the tunnelling rate given by Eq. (11) for the time specified by Eq (12).

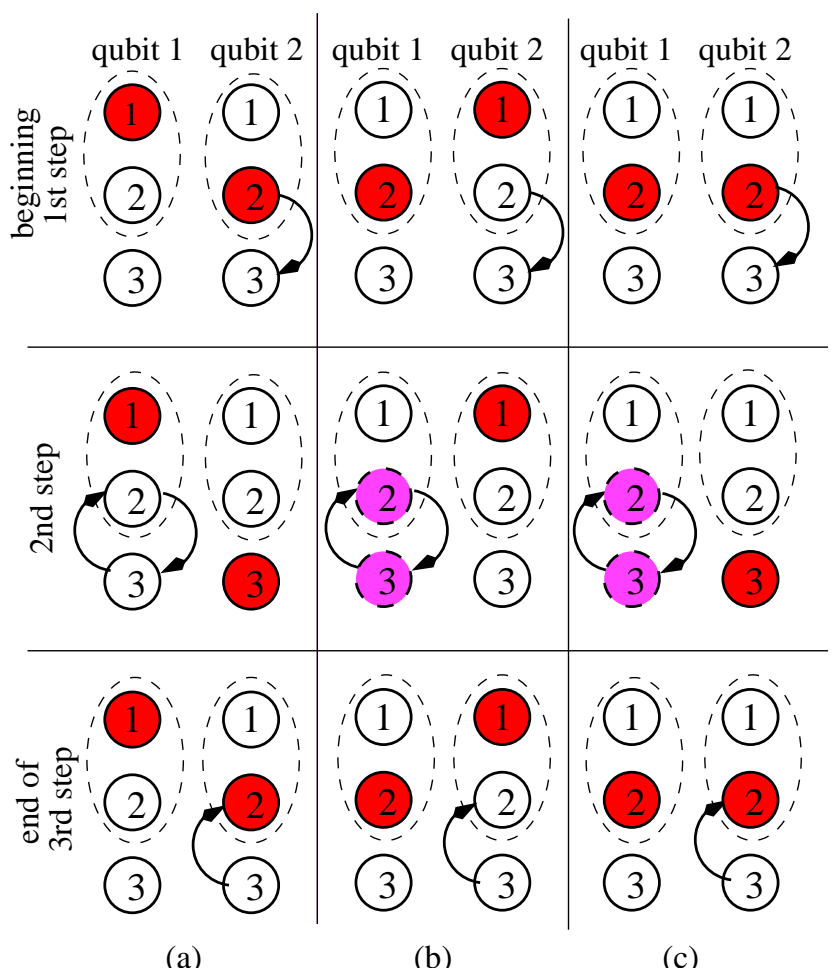

FIG. 4: Schematic representation of the phase gate operation for three initial basis states (a) $|12\rangle$, (b) $|21\rangle$, and (c) $|22\rangle$. The operation for $|11\rangle$ has been omitted because it is trivial. The filled (red) dots show the positions of the electrons at the beginning of the first step (top), during the second step (middle), and after completion of the third step (bottom). For initial configurations $|21\rangle$ (b) and $|22\rangle$ (c), the first charge is in a superposition of states $|2\rangle_{1}$ and $|3\rangle_{1}$ during the second step, and hence acquires a phase conditional on the population of state $|3\rangle_{2}$, which is indicated by by lighter (pink) shading. Notice, however, that the electron starts and ends in state $|2\rangle_{1}$, except for the conditional phase acquired.

3. Acting on the second qubit, repeat the first step to swap the populations of states $|2\rangle_{2}$ and $|3\rangle_{2}$ back.

4. Acting simultaneously on both qubits, shift the energy of states $|2\rangle_{1}$ and $|2\rangle_{2}$ by $\epsilon_{2}^{(1)}=\ell \pi / \tau_{4}$ and $\epsilon_{2}^{(2)}=\pi / \tau_{4}$, respectively, for some time $\tau_{4}$, where $\ell=1 / 2-(n+k) \bmod 2$. (See appendix $[$ )

The first three steps are illustrated in Fig. 4 and Fig. 5 shows results for a simulation of the gate for ideal pulses and no constraints.

Since this gate combined with local unitary operations as described in Sec. III is universal, we can implement any desired two-qubit gate. For instance, a controlledNOT gate is performed simply by applying a Hadamard transformation on the second qubit before and after the pulse sequence above.

Further analysis shows that the conditional phase acquired by state $|2\rangle_{1}$ is exactly twice the geometric phase acquired by the two-level subsystem $S_{1}=\{|21\rangle,|31\rangle\}$ 


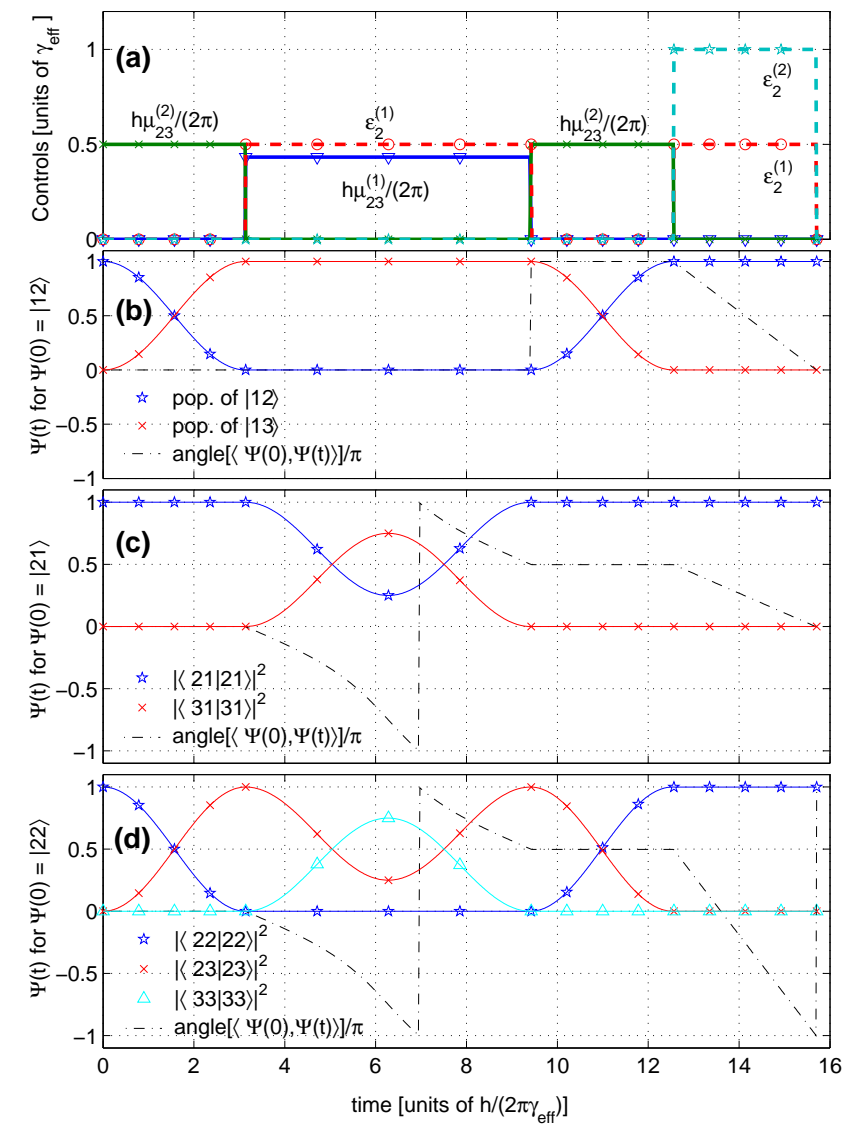

FIG. 5: Operation of the proposed controlled phase gate for ideal controls with no constraints. Shown are the control parameter settings (a) and the corresponding evolution of the initial states (b) $|12\rangle$, (c) $|21\rangle$ and (d) $|22\rangle$. The evolution of $|11\rangle$, being trivial, has been omitted. In each case, only the relevant populations and acquired phases are plotted.

and $S_{2}=\{|23\rangle,|33\rangle\}$, respectively. To see this, note that the Hamiltonians for $S_{1}$ and $S_{2}$ are

$$
\begin{aligned}
& \hat{H}_{S_{1}}=\frac{1}{4} \hat{I}_{2}+\mu \hat{\sigma}_{x}+\frac{1}{4} \hat{\sigma}_{z} \\
& \hat{H}_{S_{2}}=\frac{3}{4} \hat{I}_{2}+\mu \hat{\sigma}_{x}-\frac{1}{4} \hat{\sigma}_{z}
\end{aligned}
$$

with $\mu=\left(\sqrt{(2 n)^{2} /(2 k-1)^{2}-1}\right) / 4$, and we can visualize their evolution on the Bloch sphere as in Fig. 6. Let $\mathbf{s}=\left(s_{x}, s_{y}, s_{z}\right)$ with $s_{\alpha}=\operatorname{Tr}\left(\sigma_{\alpha} \rho\right)$ for $\alpha \in\{x, y, z\}$ be the usual Bloch vector. The initial states $|21\rangle$ and $|23\rangle$ for $S_{1}$ and $S_{2}$, respectively, correspond to the Bloch vector $\mathbf{s}_{0}=(0,0,1)$, and their evolution in $\mathbb{R}^{3}$ to a rotation about the axes $\mathbf{d}_{1}=2(\mu, 0,1 / 4)$ and $\mathbf{d}_{2}=2(\mu, 0,-1 / 4)$, respectively.

The pure-state non-adiabatic, cyclic geometric phase [26] is $\phi_{\text {geom }}=\frac{\Omega}{2}=2 \pi n(1-\cos \theta)$, where $\Omega$ is the solid angle subtended by the Bloch vector, $n$ is the number of times the Bloch vector rotates around the axis $\mathbf{d}$, and $\theta$ is the angle between the initial state vector $\mathbf{s}_{0}$ and the rotation axis $\mathbf{d}$. Both states rotate with the same

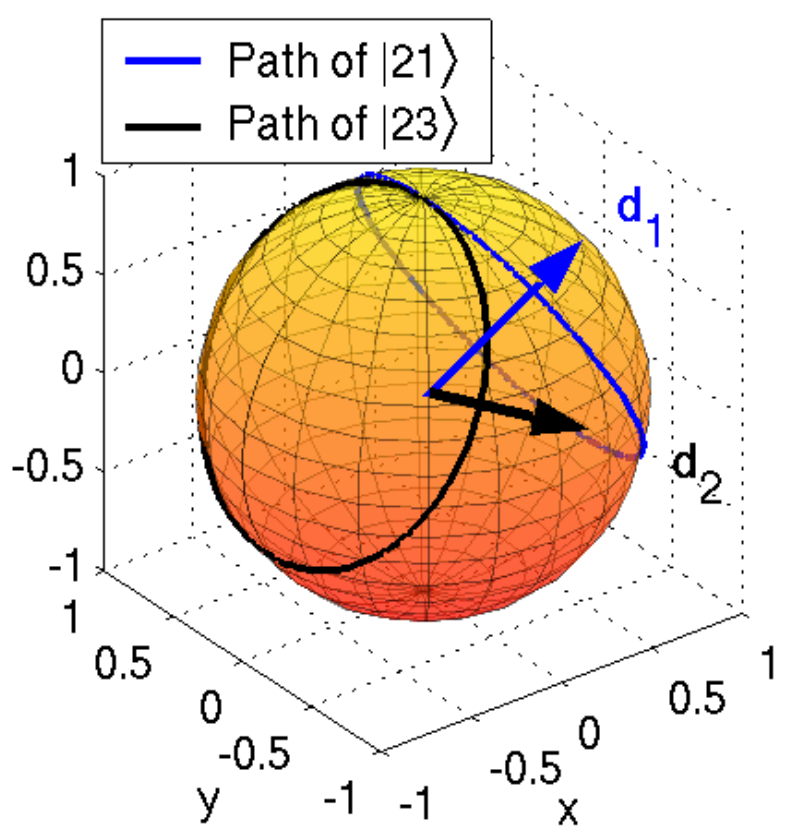

FIG. 6: Trajectory of the Bloch vectors associated with the two-level subsystems $S_{1}$ and $S_{2}$ for ideal phase gate. For $n=k=1$ the Bloch vectors $\mathbf{s}_{1}(t)$ (cyan) and $s_{2}(t)$ (black) rotate about $\mathbf{d}_{1}$ and $\mathbf{d}_{2}$, respectively, simultaneously completing a single closed loop on the surface of the Bloch sphere and sweeping out solid angles of $\pi$ (area "inside" the blue loop) and $3 \pi$ (area "outside" the black loop), respectively. Hence, the difference between the areas is $2 \pi$, and the conditional phase acquired by state $|2\rangle_{1}$ depending on whether state $|3\rangle_{2}$ is occupied will differ by $\pi$.

frequency $\|\mathbf{d}\|=\left\|\mathbf{d}^{\prime}\right\|=\left(\sqrt{1+16 \mu^{2}}\right) / 2=n /(2 k-1)$, and hence the relative geometric phase depends on $\theta$. Noting that

$$
\cos \left(\theta_{1}\right)=\frac{\mathbf{d}_{1} \cdot \mathbf{s}_{0}}{\left\|\mathbf{d}_{1}\right\|}=\frac{2 k-1}{2 n}=-\cos \left(\theta_{2}\right),
$$

we see that the respective geometric phases acquired by $S_{1}$ and $S_{2}$ are $2 \pi n[1-(2 k-1) /(2 n)]=\pi[2 n-(2 k-1)]$ and $2 \pi n[1+(2 k-1) /(2 n)]=\pi[2 n+(2 k-1)]$, i.e., exactly twice the conditional phases acquired by states $|21\rangle$ and $|23\rangle$.

\section{REALISTIC CONTROLS AND CORRECTION OF SYSTEMATIC ERRORS}

So far we have assumed ideal, piecewise-constant control Hamiltonians. In reality, however, the pulses will have finite rise .. and fall times, for example, as shown in Fig. 17. A naïve implementation of the above scheme would perforce introduce systematic errors. These errors are evident in Fig. 8 (I), which show the evolution of the system for the same parameter values as in Fig. 15 except 


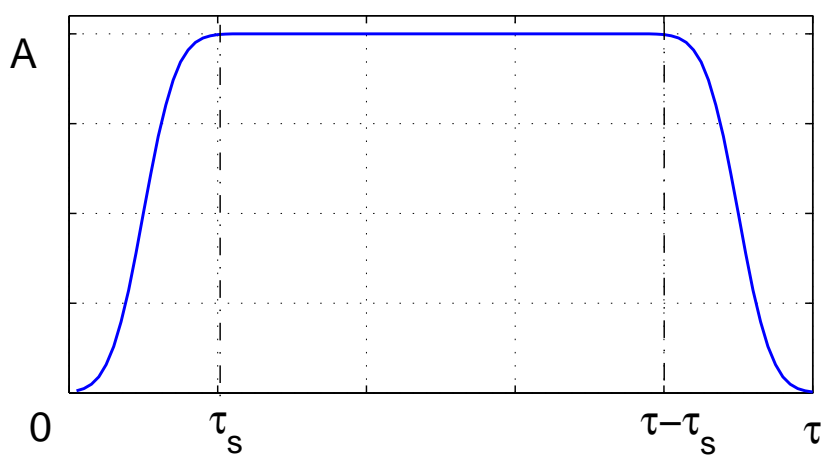

FIG. 7: Realistic square-wave pulse of amplitude $A$ and length $\tau$ with rise and decay time $\tau_{s}$, modelled using error functions.

\begin{tabular}{|r|c|c|c|c|c|c|}
\hline$\tau_{s}$ & 0.25 & 0.50 & 0.75 & 1.00 & 1.25 & 1.50 \\
\hline $\mathcal{E}_{u}$ & 0.0223 & 0.0825 & 0.1691 & 0.2659 & 0.3559 & 0.4251 \\
\hline $\mathcal{E}_{c} \times 10^{4}$ & 0.2225 & 0.2249 & 0.2273 & 0.2294 & 0.2311 & 0.2325 \\
\hline
\end{tabular}

TABLE I: Gate error $\mathcal{E}=1-\mathcal{F}$ as a function of the pulse rise and decay time $\tau_{s}$ for non-ideal, uncorrected $\left(\mathcal{E}_{u}\right)$ and corrected pulses $\left(\mathcal{E}_{c}\right)$ for simulations with time step $\Delta t=$ 0.005 .

for pulse rise and decay times of $\tau_{s}=1$ time unit. Comparing the trajectories shows that there are significant population and phase errors. In particular, the populations of the states $|13\rangle,|31\rangle$ and $|33\rangle$ do not return to 0 , i.e., population is lost to the auxiliary states, a potentially difficult error to correct.

To quantitatively compare the gates implemented to the ideal gate we consider the average gate fidelity 27, 28]

$$
\mathcal{F}=\overline{\left\langle\Psi_{\text {in }}\left|\hat{U}^{\dagger} \hat{\rho}_{\text {out }} \hat{U}\right| \Psi_{\text {in }}\right\rangle}
$$

which is a measure of the overlap of the final state $\hat{\rho}_{\text {out }}$ with the desired target state $\hat{U}\left|\Psi_{\text {in }}\right\rangle$ averaged over all input states. Since $\hat{\rho}_{\text {out }}$ may extend to the auxiliary quantum dots, the fidelity includes the effect of population losses to these states.

In the example shown in Fig. 8 pulse rise and decay times of one time unit reduce the average gate fidelity from one in the ideal case to $\sim 73 \%$. Table $\llbracket$ shows that even relatively small rise and decay times of the fields tend to reduce the average gate fidelity noticeably. This increase in the gate error appears to be mainly due to population loss to the auxiliary states.

The first step toward improving the results is to realize that rise and decay times reduce the total pulse area. An ideal square-wave pulse $\left(\tau_{s}=0\right)$ of duration $\tau$ with amplitude $A$ has a pulse area of $A \tau$, while that of a similar pulse with rise and decay time $\tau_{s}<\tau / 2$ is only $A\left(\tau-\tau_{s}\right)$ [29]. Since the pulse area is an important control parameter, we must therefore adjust either the field strengths or the pulse lengths, or possibly both. For the 1st, 3rd and 4th step of the CPHASE gate correcting the pulse area is all that is needed to eliminate systematic errors due to non-zero pulse rise and decay times, and we can achieve this either by increasing the field strength or the pulse durations.

During the crucial 2nd step, the energy of state $|2\rangle_{1}$ should be kept constant $\epsilon_{2}^{(1)}=1 / 2$ while tunnelling is enabled, and the value of the tunnelling rate $\mu_{23}^{(1)}$ should be close to one of the desired values. Increasing the field strengths to compensate for rise and decay times is thus not an option for this step. For simplicity, we shall therefore correct the pulse areas by increasing the duration of each pulse by $\tau_{s}$, i.e., setting $\tau_{k}^{\prime}=\tau_{k}+\tau_{s}$, where $\tau_{k}$ is the duration of the corresponding ideal pulse.

To improve the accuracy of the 2 nd step further, we raise the energy $\epsilon_{2}^{(1)}$ at least $\tau_{s}$ time units before we enable tunnelling between states $|2\rangle_{1}$ and $|3\rangle_{1}$, and lower it only after the tunnelling barrier has been raised again to inhibit tunnelling. Since changing the energy of state $|2\rangle_{1}$ is a local operation on the first qubit, it commutes with the swap operation on states $|2\rangle_{2}$ and $|3\rangle_{2}$. We can therefore begin to raise the energy of state $|2\rangle_{1}$ before the 1st swap operation is completed, and we can begin the 3rd swap operation before $\epsilon_{2}^{(1)}(t)$ has reached zero. However, the increased duration of the energy shift will induce an additional local phase shift, which has to be taken into account in the final step to achieve the desired gate. Let $t_{1}=\tau_{1}^{\prime}-\tau_{s}$ and $t_{2}=\tau_{1}^{\prime}+\tau_{2}^{\prime}+\tau_{s}$ and

$$
\Delta \phi=\int_{t_{1}}^{t_{2}} \epsilon_{2}^{(1)}(t) d t-\frac{1}{2} \tau_{2},
$$

where $\tau_{2}$ is the time required to complete the second swap operation for ideal, piecewise constant control pulses. Then the local phase rotation on the first qubit required in the final step is $\Phi=\ell \pi-\Delta \phi$ instead of $\ell \pi$, where $\ell=\frac{1}{2}-(n+k) \bmod 2$ as before. Thus, in the final step the energy of state $|2\rangle_{1}$ must be raised by

$$
\epsilon_{2}^{(1)}=\frac{\pi \ell-\Delta \phi}{\tau_{4}}
$$

where $\tau_{4}$ is the duration of the step for ideal, piecewise constant controls.

Numerical simulations indicate that these corrections greatly enhance the performance of the phase gate. For instance, Table \ shows that the average gate error $\mathcal{E}_{c}$ with these corrections is less than $10^{-4}$ for all rise and decay times $\tau_{s}$ [33] and Fig. [8 (II) shows that the trajectories very closely match those of the ideal gate.

\section{GATE OPERATION TIMES AND PHYSICAL CONSTRAINTS}

In the hypothetical case of no constraints on the tunnelling rates, energy shifts and pulse lengths considered so far, the first and third swap operation as well as the local phase rotations could, in principle, be implemented 
(I)
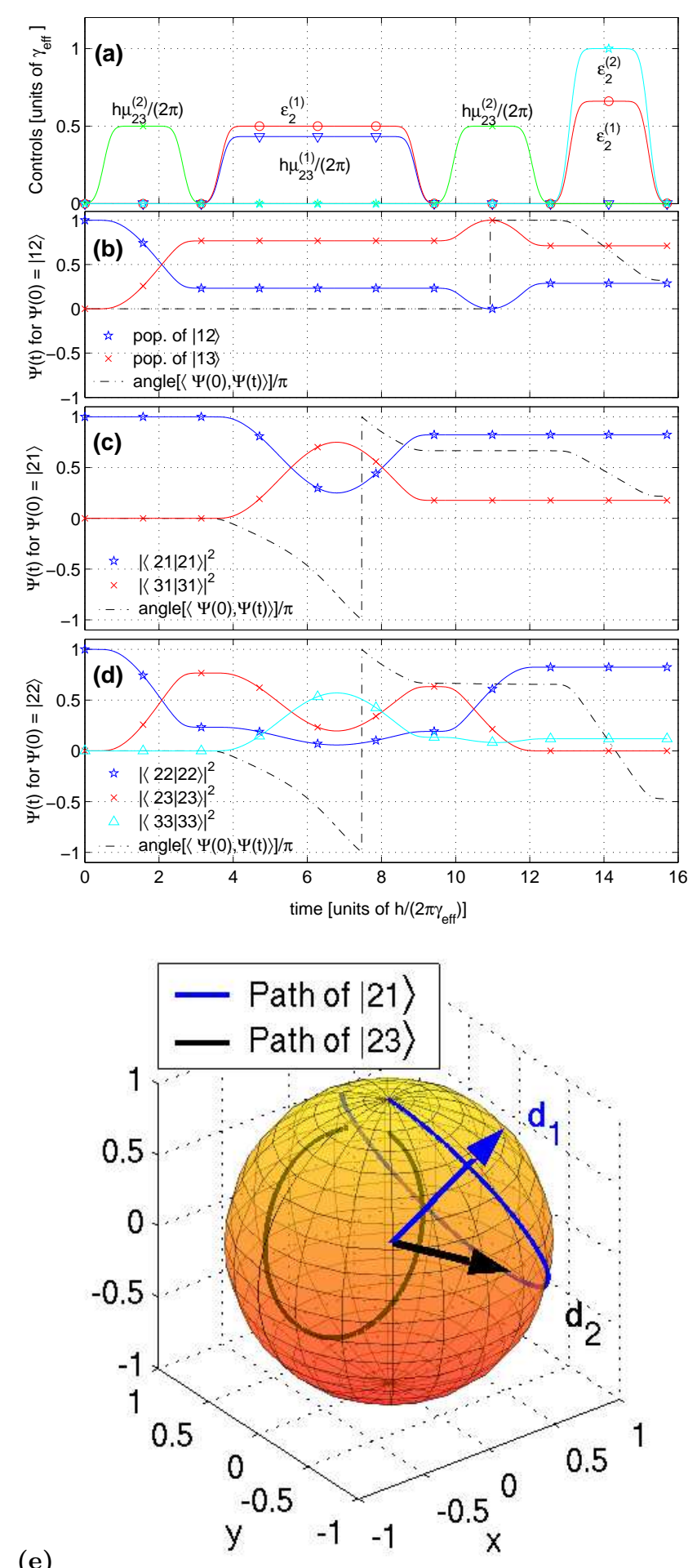

(II)
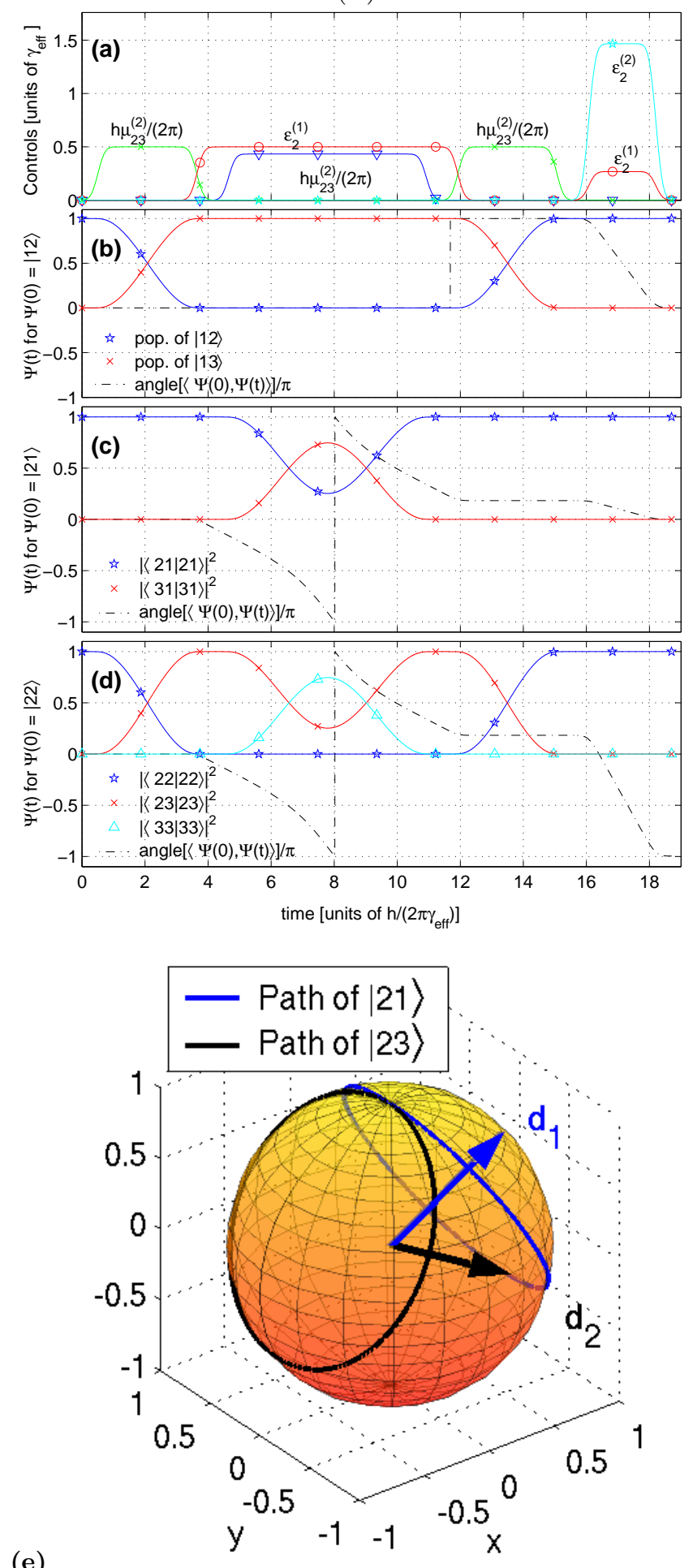

(e)

FIG. 8: Control parameter settings (a) and evolution of the initial states (b) $|12\rangle$, (c) $|21\rangle$ and (d) $|22\rangle$, as well as (e) evolution of the two-level subsystems $S_{1}$ and $S_{2}$ on the Bloch sphere under the operation of the controlled phase gate $(n=k=1)$ for controls with rise and decay time $\tau_{s}=1\left[\hbar / \gamma_{\text {eff }}\right]$ without correction of systematic errors (I) and with corrections described (II). Due to the rise and decay time of the controls, the trajectories of the Bloch vectors in (I) do not form closed loops, and the system does not return to its initial state (modulo the desired phase change). However, when the rise and decay times of the pulses are taken into account, population loss to the auxiliary states is negligible and the trajectories of the Bloch vectors again form closed loops (II). 
arbitrarily fast and the gate operation time would be limited mainly by the time required to complete the second step, i.e., $\tau \approx \tau_{2}=2 \pi$ time units. In reality, however, the gate operation time is usually limited by physical and technical constraints that impose, for instance, a minimum pulse length $\tau_{\min }$ and maximum tunnelling rate $\mu_{\max }$ between the qubit and auxiliary quantum dot.

To explore the consequences of finite tunnelling rates and switching speeds, let us consider a specific example of a 2D charge qubit architecture as shown in Fig. 2 (a) with auxiliary quantum dots spaced about $2 a=170 \mathrm{~nm}$ apart [34]. Neglecting screening effects, the Coulomb energy in this case is $\gamma_{\mathrm{eff}} \approx 0.718 \mathrm{meV}$, and the characteristic time scale $\hbar \gamma_{\text {eff }}^{-1} \approx 1$ ps [35] Thus, theoretically, two-qubit gate operation times of less then 20 ps could be achieved, as the previous sections show, and if one assumes that local operations can be realized arbitrarily fast then gate operation times of less than 7 ps would be possible.

However, if the pulse lengths must be $\gtrsim 50 \mathrm{ps}-$ about the limit of what is accessible with current technology - then Eq. (E1) shows that the best possible choice of the parameters $n$ and $k$ that satisfies this constraint while minimizing the gate operation time is $k=n=5$. The total gate operation time in this case increases to $3 \times 50+18 \pi \approx 206.55$ ps - assuming we can achieve the required tunnelling rate of $\mu_{23}^{(1)}=(1 / 4) \sqrt{(10 / 9)^{2}-1}\left[\gamma_{\mathrm{eff}} / \hbar\right] \approx 1.21 \times 10^{11} \mathrm{rad} \mathrm{s}^{-1}$. The evolution of the system for the resulting gate is shown in Fig. 9 (I).

If the maximum tunnelling rate that can be achieved (e.g., without applying control voltages that might lead to a breakdown of the oxide layer separating the silicon substrate and the surface control electrodes, or pulses that might result in population loss to higher-lying excited states) are lower than this, then the gate operation time will be increased further. For example, if we must satisfy $\mu_{23}^{(1)} \leq 10^{-11} \mathrm{rad} \mathrm{s}^{-1}$ then Eqs. (E4), (E5) show that the best choice for $n$ and $k$ is $n=k=7$, which yields $\mu_{23}^{(1)} \approx 0.99 \times 10^{11} \mathrm{rad} \mathrm{s}^{-1}$, and the gate operation time increases to 231.68 ps, as shown in Fig. 9 (II).

\section{GATE ROBUSTNESS FOR NOISY CONTROLS}

Another important issue in practice is the robustness of the gate for noisy controls. To study this effect we artificially added noise to our controls. Fig. [10 shows the increase of the gate error $\mathcal{E}$ as a function of the magnitude of the noise added. The simulations were performed with a fixed time step of $\Delta t=0.005$, for which the average gate fidelity in the absence of noise exceeded 0.9999. The noise functions $\eta(t)$ were bounded

$$
|\eta(t)| \leq \eta_{0}
$$

with Fourier transforms $\tilde{\eta}(\omega)$ satisfying

$$
|\tilde{\eta}(\omega)| \leq \begin{cases}0 & \omega=0 \\ K / \omega_{0} & \omega \leq \omega_{0} \\ K / \omega & \omega>\omega_{0}\end{cases}
$$

where $K$ is a constant. This type of noise corresponds to $1 / f$ noise with a low-frequency cut-off, which is common in electronic devices.

Although the addition of noise necessarily increase the gate error, the gate appears to be quite robust to this noise, as a typical example of the evolution of the system for noisy controls (Fig. [11) shows. Not unsurprisingly, the curves for varying threshold frequencies $\omega_{0}$ in Fig. 10 suggest that the gate is more sensitive to low-frequency than high-frequency noise, as the high-frequency components tend to cancel.

\section{GATE ROBUSTNESS FOR IMPERFECT GEOMETRIES}

A final issue that must be considered is the effect of manufacturing tolerances, which result in deviations of the qubit register from an ideal specification. In solidstate charge-based architectures of the types considered herein, the main issues appear to be imperfections in the placement or geometry of the quantum dots. The former is believed to be especially pronounced for quantum dots based on donor impurities in a Silicon matrix, while the latter will be relevant, e.g., for manufactured $\mathrm{Ga} / \mathrm{GaAs}$ heterostructure quantum dots. As modelling all the possible effects of imperfections in the fabrication process for heterostructure quantum dots would far exceed the scope of this paper, we shall focus on modelling the effect of imperfect placement of the quantum dots on the gate performance.

For solid-state architectures based on donor impurities (e.g., Phosphorus) in a Silicon matrix the accuracy of placement of the donors is limited. In a shielded $2 \mathrm{D}$ architecture inaccurate placement of the donors will mainly cause variations in the tunnelling rates as the structure of the silicon lattice introduces spatial oscillations in the donor wavefunctions, and hence tunnelling rates 30] analogous to those seen in exchange systems 31, [32]. There will also be minor changes in the strength of the Coulomb interaction between the auxiliary sites. However, the actual Coulomb coupling strengths and tunnelling rates as a function of the control voltages applied can, in principle, be determined using Hamiltonian identification techniques similar to those described in Ref. [19]), for instance, and the control scheme can then easily be adapted to achieve the desired gate for the actual system.

The situation is different for 3D architectures [cf. Fig. 2 (b)] that rely on symmetry to effectively cancel the Coulomb interactions between the qubit sites. Manufacturing tolerances in this case will result in asymmetries of 
(I)
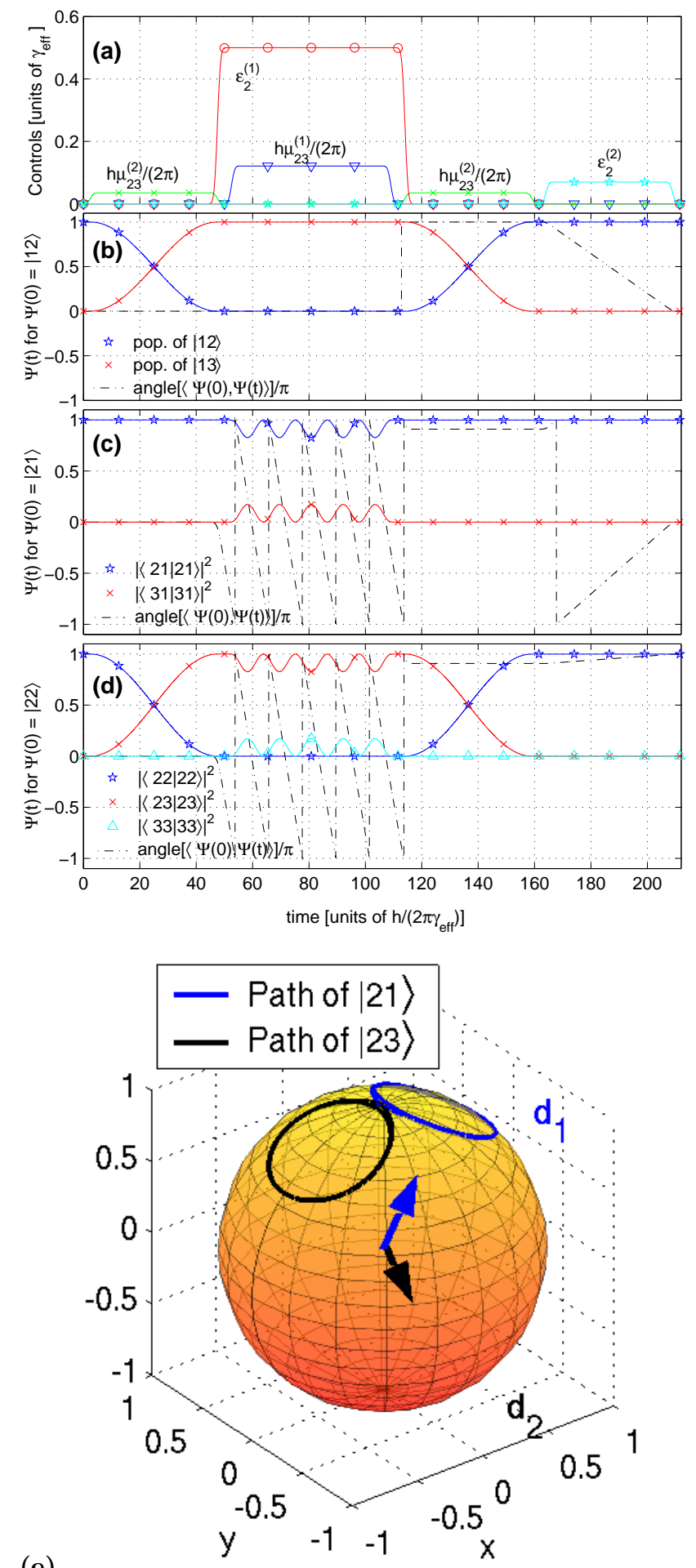

(II)
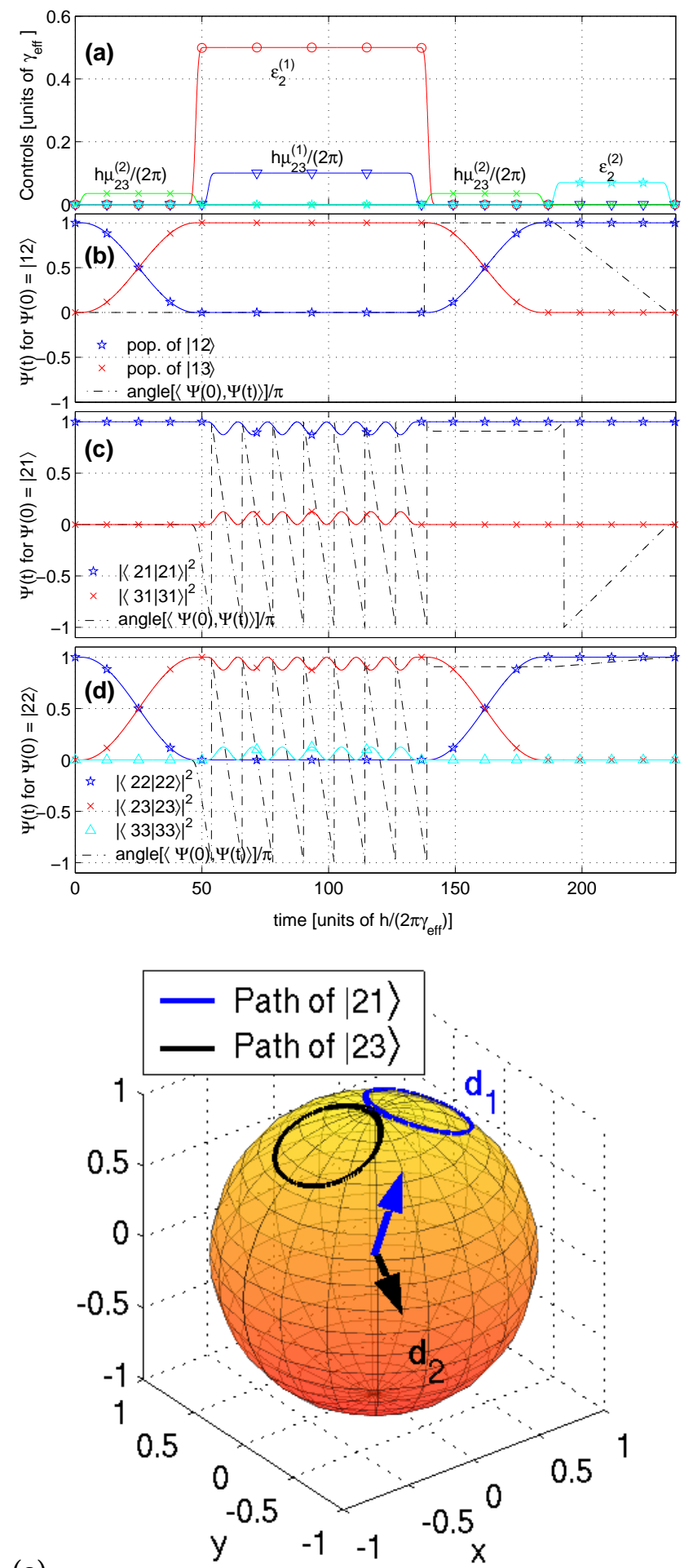

FIG. 9: Control settings (a) and corresponding evolution of the initial states (b) $|12\rangle$, (c) $|21\rangle$, and (d) $|22\rangle$, as well as evolution of the two-level subsystems $S$ and $S^{\prime}$ on the Bloch sphere (e) under the operation of the controlled phase gate when (I) minimum pulse length constraints of $\tau_{\min } \geq 50$ ps necessitate $n=k=5$, and (II) simultaneous pulse length and tunnelling rate constraints $\tau_{\min } \geq 50 \mathrm{ps}$ and $\mu_{23}^{(1)} \leq 10^{-11} \mathrm{rad} \mathrm{s}^{-1}$ necessitate $n=k=7$. Note the decrease in the angle $\theta_{1}$ (increase in $\theta_{2}$ ) between the rotation axis $\mathbf{d}_{1}\left(\mathbf{d}_{2}\right)$ and the (positive) $z$-axis compared to the $n=k=1$ case, and the resulting greater difference in the areas swept out by the Bloch vectors $\mathbf{s}_{1}$ (blue) and $\mathbf{s}_{2}$ (black) in a single loop: $\pi / 5$ versus $19 \pi / 5$ in (I); $\pi / 7$ versus $27 \pi / 7$ in (II). Consequently, five and seven loops, respectively, are necessary to archive an area difference that is a multiple of $2 \pi$, and the time required to implement the phase shift increases to $18 \pi$ and $26 \pi$, respectively. Also note the slight distortion of the loops near the north pole due to the dynamic change of the rotation axis during rise and decay periods of the pulses. Although present for all choices of $n$ and $k$, it is more pronounced for larger $n$ and $k$. 


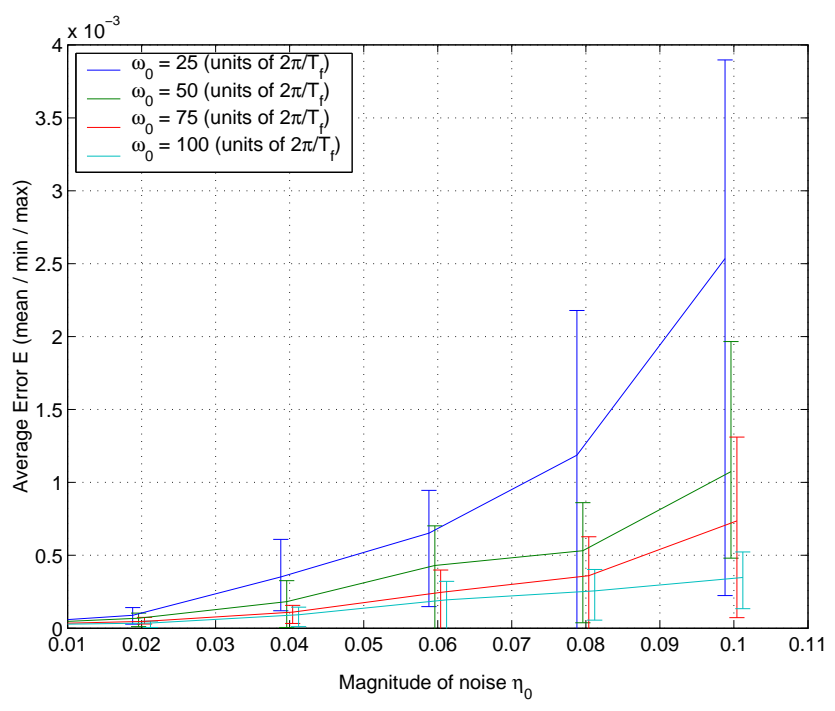

FIG. 10: Average gate error $\mathcal{E}$ as a function of the magnitude $\eta_{0}$ of the noise for various threshold frequencies $\omega_{0}$. The solid curves show the mean of $\mathcal{E}$ and the error bars indicate the range of $\mathcal{E}$ over 10 simulations. The data were plotted horizontally offset to improve visual clarity.

the geometry, which cannot be compensated easily, even if they could be identified precisely, and perforce reduce the gate fidelity. To estimate the effect of such errors we have performed computer simulations. For a 3D arrangement of six quantum dots as shown in Fig. 2(b), we randomly perturbed the positions of all six quantum dots by up to four lattice sites in the $x$ - and $y$-directions, and \pm 1 monolayer in the $z$-direction, assuming a lattice constant of $0.3 \mathrm{~nm}$, values that appear feasible with current fabrication technology [23], and numerically computed the average gate fidelity $\mathcal{F}$ for each perturbed system. Note that it was assumed here that the tunnelling rates can be kept steady despite the effect of the silicon lattice by adjusting the control voltages appropriately.

The numerical results in Table【 suggest that the robustness of our phase gate with respect to asymmetries depends significantly on the choice of interqubit spacings and the distance between the auxiliary sites. Concretely, the data suggest that the robustness of the gate with respect to random pertubations of the geometry is maximized by minimizing the distance between auxiliary sites while maximizing the distances between qubits. There also appears to be a strong relation between the robustness with respect to asymmetries and the effective Coulomb interaction between the auxiliary sites in the system, suggesting that maximizing the later quantity might be advantageous. However, it should be noted that stronger Coulomb coupling implies shorter gate operation times unless the gate parameters are changed, which might affect the gate fidelity. A final computation for a (target) geometry with $a=20, b=100$ and $c=10$ showed that the average gate fidelity over 100 random perturbations ranged from 0.9944 to 1.0000 with
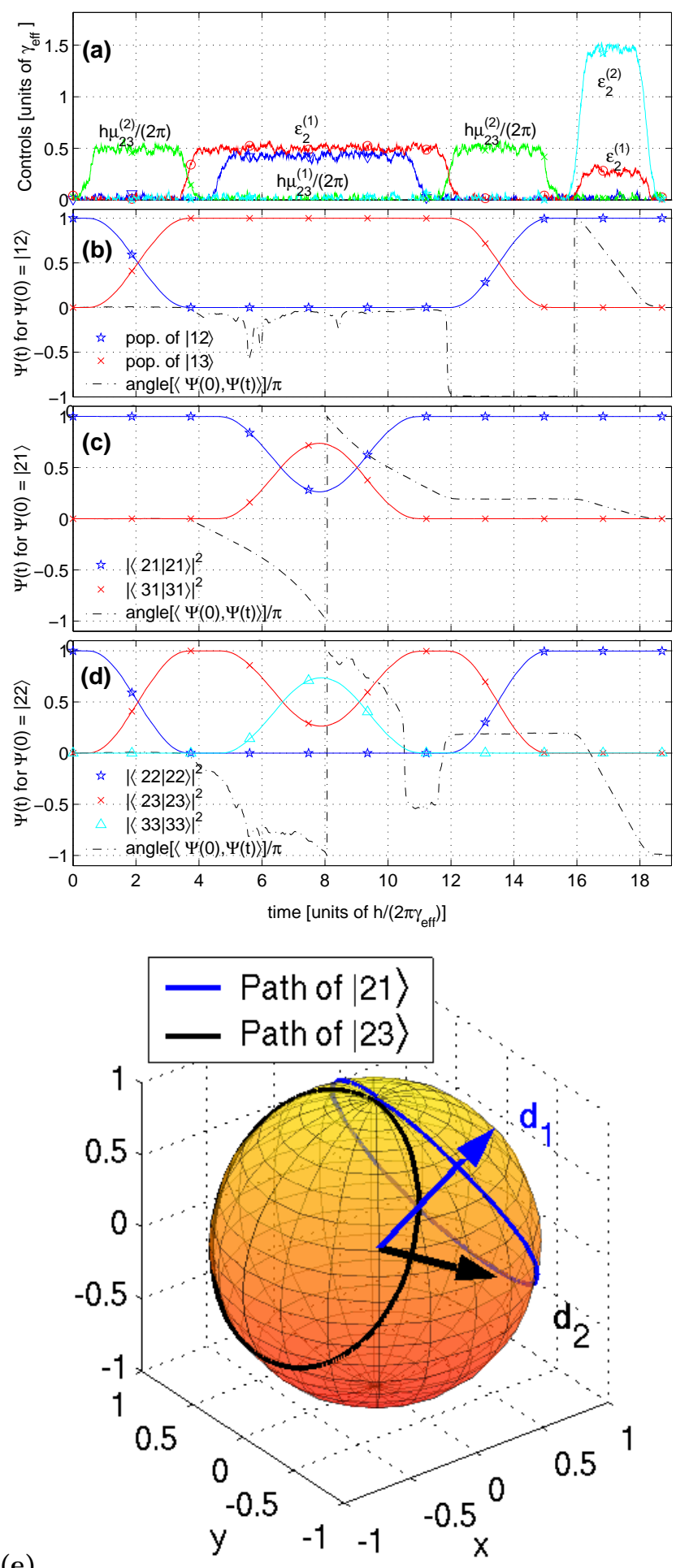

(e)

FIG. 11: Control settings (a) and corresponding evolution of the initial states (b) $|12\rangle$, (c) $|21\rangle$ and (d) $|22\rangle$, as well as evolution of the two-level subsystems $S_{1}$ and $S_{2}$ on the Bloch sphere (e) under the operation of our phase gate for noisy controls $\left(\eta_{0}=0.1, \omega_{0}=50\right)$. The noise has no noticable effect on the evolution of the populations. The relative phases exhibit some wiggles but they mostly seem to average out over the duration of the gate. The average gate error was $\mathcal{E}=0.708 \times 10^{-3}$. 


\begin{tabular}{|c|c|c|c|c|}
\hline $\mathrm{a} \backslash \mathrm{b}$ & 30 & 50 & 70 & 90 \\
\hline 20 & 0.964022 & 0.996631 & 0.998153 & 0.998855 \\
\hline 40 & N/A & 0.909071 & 0.992692 & 0.998410 \\
\hline 60 & N/A & N/A & 0.831827 & 0.989618 \\
\hline 80 & N/A & N/A & N/A & 0.808333 \\
\hline
\end{tabular}

TABLE II: Mean of the average gate fidelity $\mathcal{F}$ for different geometries with fixed intra-qubit spacing of $c=10 \mathrm{~nm}$. For each target geometry the mean of $\mathcal{F}$ was computed for 30 randomly perturbed systems. The gate parameters for all simulations were $n=k=1$ and the time steps were chosen such that the average gate fidelity of each unperturbed systems was $\geq 0.99995$. Notice that the mean of $\mathcal{F}$ increases sharply for decreasing values of $a$, and noticably for increasing values of $b$.

a mean (standard deviation) of 0.9989 (0.0013). These results suggest that even $3 \mathrm{D}$ charge-qubit architectures without shielding could be designed to be rather robust with respect to fabrication errors.

\section{CONCLUSIONS}

We have presented two scalable achitectures for solidstate charge qubits that permit controlled entangling operations between pairs of qubits. We add that these operations could also be performed in parallel for cluster state preparation, a concept that we will explore elsewhere. The key feature of both geometries is that interactions between qubits are mediated via auxiliary quantum dots, while direct interactions between qubits are suppressed either through the use of shielding, or a threedimensional design to cancel interactions. Controlled entangling gates can therefore be implemented by switching the charge distribution, and hence the Coulomb interaction, between qubits using the auxiliary dots. Both systems should be realizable using existing or near-future fabrication techniques.

In particular, we have shown explicitly how to realize a controlled phase gate, i.e., a universal, maximally entangling two-qubit gate by a simple four-step procedure. The crucial step in the sequence is the controlled tunnelling between a qubit state and an auxiliary dot, which induces a phase shift conditional on the occupation of an adjacent (auxiliary) quantum dot. The scheme is sufficiently flexible to accommodate practical constraints on both pulse lengths and tunnelling rates. Analysis of the gate operation shows that the controlled phase shift can be explained in terms of dynamic and geometric phases. Due to the strength of the Coulomb coupling the gate operation is surprisingly fast. In the absence of pulse length constraints gate operation times on the order of a few picoseconds would be theoretically possible, and even with currently realistic constraints on the pulse lengths and tunnelling rates, gate operation times around $200 \mathrm{ps}$ should be attainable.

Using computer simulations, we have also studied the effects of systematic errors on the gate performance. The simulations show that finite pulse rise and decay times tend to result in population loss to the auxiliary states, and can noticeably reduce the average gate fidelity. The average gate error increases sharply with increasing pulse rise and decay times. The ideal scheme, however, can easily be generalized to compensate for such systematic errors. Simulations show that these corrections can greatly improve the average gate fidelity; the corrected pulse sequences always achieved average gate fidelities of $>99.99 \%$, and in some cases the average gate fidelity increased from $\sim 70 \%$ without corrections to $>99.99 \%$. These results suggest that (experimental) characterization of the rise and decay times of the pulses is very important to achieve high gate fidelity.

We have also performed simulations to assess the robustness of the gate to noisy control pulses. The results suggest that the gate is quite robust with regard to both bandwith-limited $1 / f$ noise and white noise. High-frequency noise tends to effectively cancel. Lowfrequency fluctuations of the control pulses can reduce the gate fidelity. Surprisingly, however, the resulting average gate errors are generally very small compared to the systematic errors. In most of our simulations, the average gate error increased only from $<10^{-4}$ to $<10^{-3}$, even for very noisy controls. Our simulations further suggest that even a 3D geometry without shielding, which relies mainly on symmetry to cancel the effect of the Coulomb interaction between qubits, can be made to be quite robust to misalignment errors during fabrication if the parameters of the geometry are chosen sufficiently carefully.

\section{Acknowledgments}

The authors thank C. J. Wellard (Univ. of Melbourne) for useful discussions. S.G.S and D.K.L.O acknowledge financial support from the Cambridge-MIT Institute, Fujitsu and IST grants RESQ (IST-2001-37559) and TOPQIP (IST-2001-39215). S.G.S also acknowledges support from the EPSRC IRC in quantum information processing, and D.K.L.O thanks Sidney Sussex College for support. A.D.G is supported by the Australian Research Council, the Australian government and the US National Security Agency (NSA), Advanced Research and Development Activity (ARDA) and the Army Research Office (ARO) under contract number DAAD1901-1-0653, and acknowledges support from a Fujitsu visiting fellowship while visiting the University of Cambridge.

\section{APPENDIX A: LOCAL OPERATIONS}

We observed in Sec.IIthat any local unitary operation can be realized by concatenating three elementary rotations. It is worth noting, however, that some local unitary operators can be implemented more efficiently if we allow simultaneous changes of more than one control pa- 
rameter such as the energy difference $\Delta \epsilon_{12}=\left(\epsilon_{2}-\epsilon_{1}\right) / 2$ and the tunnelling rate $\mu_{12}$ between states $|1\rangle$ and $|2\rangle$. To see this note that we can rewrite Eq. (2) as follows:

$$
\hat{H}\left(\epsilon_{d}, \mu_{12}\right)=\bar{\epsilon}_{12} \hat{I}_{12}+\Delta \epsilon_{12} \hat{Z}_{12}+\hbar \mu_{12} \hat{X}_{12}+\epsilon_{3}|3\rangle\langle 3|
$$

where $\bar{\epsilon}_{12}=\left(\epsilon_{1}+\epsilon_{2}\right) / 2$ and $\hat{Z}_{12}=|2\rangle\langle 2|-| 1\rangle\langle 1|$. Thus, if we apply constant energy shifts $\epsilon_{d}$ and effect a fixed tunnelling rate $\mu_{12}$ between dots 1 and 2 (while all other tunnelling rates are kept zero) for $0 \leq t^{\prime} \leq t$ then we generate the unitary operator:

$$
\begin{aligned}
& \exp \left[-i t \hat{H}\left(\epsilon_{d}, \mu_{12}\right) / \hbar\right] \\
= & {\left[\cos (\alpha t) \hat{I}_{12}-i \frac{\sin (\alpha t)}{\alpha \hbar}\left(\Delta \epsilon_{12} \hat{Z}_{12}+\hbar \mu_{12} \hat{X}_{12}\right)\right] \times } \\
& \exp \left(-i t \bar{\epsilon}_{12} / \hbar\right)+\exp \left(-i t \epsilon_{3} / \hbar\right)|3\rangle\langle 3|
\end{aligned}
$$

where $\alpha=\sqrt{\left(\Delta \epsilon_{12} / \hbar\right)^{2}+\mu_{12}^{2}}$. Thus, we can realize a Hadamard transform, for instance, in a single step by setting $\Delta \epsilon_{12}=-\hbar \mu_{12} \neq 0$ for time $t=\pi /\left(2 \sqrt{2} \mu_{12}\right)$.

\section{APPENDIX B: FIRST SWAP OPERATION}

The total Hamiltonian for the first swap operation is $\hat{H}_{1}=\hat{H}_{0}+I_{3} \otimes \hat{H}^{(2)}$ where

$$
\hat{H}^{(2)}=\hbar \mu_{23}^{(2)}(|2\rangle\langle 3|+| 3\rangle\langle 2|)
$$

and $\hat{H}_{0}=|33\rangle\langle 33|$ as before. Applying this Hamiltonian for time $0 \leq t^{\prime} \leq t$ gives rise to the unitary operator $\hat{U}_{1}(t)=\exp \left[-(i t / \hbar) \hat{H}_{1}\right]$. If we set $t=\tau_{1}=\pi /\left(2 \mu_{23}^{(2)}\right)$ we obtain the block-diagonal matrix

$$
\hat{U}\left(\tau_{1}\right)=\operatorname{diag}(1,-i X, 1,-i X, 1, W)
$$

where $X=\left(\begin{array}{ll}0 & 1 \\ 1 & 0\end{array}\right)$ and $W$ is a symmetric $2 \times 2$ matrix with

$$
\begin{aligned}
& W_{11}=e^{-i \tau_{1} / 2}\left[\cos \left(\frac{\tau_{1} u_{1}}{2}\right)+\frac{i}{u_{1}} \sin \left(\frac{\tau_{1} u_{1}}{2}\right)\right] \\
& W_{12}=e^{-i \tau_{1} / 2}\left[-2 i \frac{\mu_{23}^{(2)}}{u_{1}} \sin \left(\frac{\tau_{1} u_{1}}{2}\right)\right] \\
& W_{22}=e^{-i \tau_{1} / 2}\left[\cos \left(\frac{\tau_{1} u_{1}}{2}\right)-\frac{i}{u_{1}} \sin \left(\frac{\tau_{1} u_{1}}{2}\right)\right]
\end{aligned}
$$

and $u_{1}=\sqrt{1+4\left[\mu_{23}^{(2)}\right]^{2}} . \quad \hat{U}_{1}\left(t_{1}\right)$ swaps the population of states $|2\rangle_{2}$ and $|3\rangle_{2}$ provided that state $|3\rangle_{1}$ is not occupied.

\section{APPENDIX C: SECOND SWAP OPERATION}

The total Hamiltonian for the second gate is $\hat{H}_{2}=$ $\hat{H}_{0}+\hat{H}^{(1)} \otimes I_{3}$ where

$$
\hat{H}^{(1)}=\frac{1}{2}|2\rangle\langle 2|+\hbar \mu_{23}^{(1)}(|2\rangle\langle 3|+| 3\rangle\langle 2|)
$$

and $\hat{H}_{0}=|33\rangle\langle 33|$ as before. Applying this Hamiltonian for time $0 \leq t^{\prime} \leq t$ gives rise to the unitary operator $\hat{U}_{2}(t)=\exp \left[-(i t / \hbar) \hat{H}_{2}\right]$, which has the general form

$$
\hat{U}_{2}(t)=\left(\begin{array}{c|cc}
I_{3} & 0 & 0 \\
\hline 0 & A & B \\
0 & B & C
\end{array}\right)
$$

where $A=\operatorname{diag}\left(a, a, a^{\prime}\right), B=\operatorname{diag}\left(b, b, b^{\prime}\right)$ and $C=$ $\operatorname{diag}\left(c, c, c^{\prime}\right)$. We must choose the gate operation time $\tau_{2}$ such that $B=0$, i.e., $b=b^{\prime}=0$. Since

$$
\begin{aligned}
b & =-4 i \exp \left(\frac{-i t}{4}\right) \frac{\mu_{23}^{(1)}}{u_{2}} \sin \left(\frac{u_{2} t}{4}\right) \\
b^{\prime} & =-4 i \exp \left(\frac{-3 i t}{4}\right) \frac{\mu_{23}^{(1)}}{u_{2}} \sin \left(\frac{u_{2} t}{4}\right)
\end{aligned}
$$

with $u_{2}=\sqrt{1+16\left[\mu_{23}^{(1)}\right]^{2}}$, this is equivalent to $u_{2} T_{2}=$ $4 n \pi$ for some integer $n$, or

$$
\tau_{2}=\frac{4 \pi n}{\sqrt{1+16\left[\mu_{23}^{(1)}\right]^{2}}} .
$$

This choice of gate operation time gives

$$
U_{2}\left(\tau_{2}\right)=\operatorname{diag}\left(1,1,1, a, a, a^{\prime}, a, a, a^{\prime}\right)
$$

where we have

$$
\begin{aligned}
a & =(-1)^{n} \exp \left(-i n \pi / u_{2}\right), \\
a^{\prime} & =(-1)^{n} \exp \left(-i 3 n \pi / u_{2}\right) .
\end{aligned}
$$

\section{APPENDIX D: LOCAL PHASE ROTATIONS}

Composing the three swap operations, noting $\hat{U}_{3}\left(\tau_{3}\right)=$ $\hat{U}_{1}\left(\tau_{1}\right)$, gives

$$
\begin{aligned}
\hat{U} & =\hat{U}_{1}\left(\tau_{1}\right) \hat{U}_{2}\left(\tau_{2}\right) \hat{U}_{1}\left(\tau_{1}\right) \\
& =\operatorname{diag}\left(1,-1,-1, a,-a^{\prime},-a, a, W \operatorname{diag}\left(a, a^{\prime}\right) W\right)
\end{aligned}
$$

whose projection onto the two-qubit subspace $\{|11\rangle,|12\rangle,|21\rangle,|22\rangle\}$ is $\tilde{U}=\operatorname{diag}\left(1,-1, a,-a^{\prime}\right)$ with $a$ and $a$ as in (C7), which is not quite a controlled phase gate yet. However, if we multiply $\tilde{U}$ by $U^{(1)} \otimes U^{(2)}$, where

$$
\begin{aligned}
& U^{(1)}=\operatorname{diag}\left(1,(-1)^{n} \exp \left(i \pi n / u_{2}\right)\right), \\
& U^{(2)}=\operatorname{diag}(1,-1)
\end{aligned}
$$

which corresponds to simultaneous local phase rotations on state $|2\rangle_{1}$ and $|2\rangle_{2}$, we obtain

$$
U^{(1)} \otimes U^{(2)} \tilde{U}=\operatorname{diag}\left(1,1,1, \exp \left(-i 2 \pi n / u_{2}\right)\right)
$$


where $u_{2}=\sqrt{1+16\left[\mu_{23}^{(1)}\right]^{2}}$ and

$$
\mu_{23}^{(1)}=\frac{1}{4} \sqrt{\left(\frac{2 n}{2 k-1}\right)^{2}-1}
$$

for positive integers $n$ and $k$ satisfying $2 n>2 k-1$. Then clearly $2 \pi n=(2 k-1) \pi u_{2}$, i.e., we have

$$
U^{(1)} \otimes U^{(2)} \tilde{U}=\operatorname{diag}(1,1,1,-1)=\hat{U}_{\text {phase }}
$$

To achieve a controlled phase gate on the qubit space we thus require

$$
\exp \left(-i 2 \pi n / u_{2}\right)=-1 \quad \Leftrightarrow \quad 2 \pi n=(2 k-1) \pi u_{2} .
$$

Substituting $u_{2}=2 n /(2 k-1)$ yields $(-1)^{n} e^{i \pi n / u_{2}}=$ $e^{i \pi(n+k-1 / 2)}$, which shows that $\hat{U}^{(1)}=\hat{U}_{2}^{(1)}(\ell \pi)$ with $\ell=$ $1 / 2-(n+k) \bmod 2$ and $\hat{U}^{(2)}=\hat{U}_{2}^{(2)}(\pi)$, i.e., we must set $\epsilon_{2}^{(1)}=\ell \pi / \tau_{4}$ and $\epsilon_{2}^{(2)}=\pi / \tau_{4}$ for some time $t=\tau_{4}$ in the final step.

\section{APPENDIX E: TUNNELLING RATE AND PULSE LENGTH CONSTRAINTS}

In absence of constraints on the tunnelling rate $\mu_{23}^{(1)}$ it is easy to see that the optimal choice of the parameters $n$ and $k$ in the second step is $k=n=1$, which implies $u_{2}=$ 2 and $\mu_{23}^{(1)}=\sqrt{3} / 4$. In practice, however, the control pulses usually cannot be arbitrarily short and there the tunnelling rates cannot be made arbitrarily large, and we can accommodate these constraints by choosing suitable values for the parameters $n$ and $k$.

If the mimimum pulse length is $\tau_{2} \geq T_{\min }$ but the tunnelling rates are unconstrained then we simply set

$$
k=\left\lceil\frac{\tau_{\min }}{4 \pi}+\frac{1}{2}\right\rceil, \quad n=k
$$

where $\lceil x\rceil$ is the smallest positive integer $\geq x$, to optimize the gate operation time while satisfying the pulse length constraint.

If we must satisfy $\mu_{23}^{(1)} \leq \mu_{\max }$ but there is no constraint on the length of the control pulses, we set

$$
\begin{aligned}
k & =\left\lfloor\left\lceil\left(u_{\max }-1\right)^{-1}\right\rceil / 2\right\rfloor+1 \\
n & =\left\lfloor(2 k-1) u_{\max } / 2\right\rfloor
\end{aligned}
$$

where $u_{\max }=\sqrt{16 \mu_{\max }^{2}+1}$ and $\lfloor x\rfloor$ is the largest (positive) integer $\leq x$, to satisfy the constraint and optimize the gate operation time.

Finally, if we must satisfy $\tau_{2} \geq \tau_{\min }$ and $\mu_{23}^{(1)} \leq \mu_{\max }$, we choose

$$
\begin{aligned}
k & =\max \left\{k_{1},\left\lceil\tau_{\min } / 4 \pi+1 / 2\right\rceil\right\} \\
n & =\left\lfloor(2 k-1) u_{\max } / 2\right\rfloor
\end{aligned}
$$

where $k_{1}=\left\lfloor\left[\left(u_{\max }-1\right)^{-1}\right\rceil / 2\right\rfloor+1$ as in (E2) and $u_{\max }=\sqrt{16 \mu_{\max }^{2}+1}$ as before.
[1] D. P. DiVincenzo, Science 270, 255 (1995).

[2] A. Barenco and et al., Phys. Rev. A 52, 3457 (1995).

[3] D. Loss and D. P. DiVincenzo, Phys. Rev. A 57, 120 (1998).

[4] B. E. Kane, Nature 393, 133 (1998).

[5] R. Vrijen et al., Phys. Rev. A 62, 012306 (2000).

[6] L. C. L. Hollenberg et al., Phys. Rev. B 69, 113301 (2004).

[7] R. G. Clark and et al., Philos. Trans. R. Soc. London, Ser. A 361, 1451 (2003).

[8] T. Schenkel et al., J. Appl. Phys. 94, 7017 (2003).

[9] A. K. Ekert and R. Jozsa, Rev. Mod. Phys. 68, 733 (1996).

[10] L. Fedichkin, M. Yanchencho, and K. A. Valiev, Nanotechnology 11, 387 (2000).

[11] T. Hayashi et al., Phys. Rev. Lett. 91, 226804 (2003).

[12] Y. Nakamura, Y. A. Pashkin, and T. S. Tsai, Nature 398, 786 (1999).

[13] M. H. Devoret and R. J. Schoelkopf, Nature 406, 1039 (2000).

[14] A. D. Greentree et al., Phys. Rev. Lett. 92, 097901 (2004).

[15] Y. A. Pashkin et al., Nature 421, 823 (2003).

[16] S. Lloyd, Science 261, 1569 (1993).

[17] S. C. Benjamin, Phys. Rev. Lett. 88, 017904 (2001).

[18] S. C. Benjamin and S. Bose, Phys. Rev. Lett. 90, 247901
(2003).

[19] S. G. Schirmer, A. Kolli, and D. K. L. Oi, Phys. Rev. A 69, 050603(R) (2004).

[20] S. Lloyd, Phys. Rev. Lett. 75, 346 (1995).

[21] W. G. van der Wiel et al., Rev. Mod. Phys. 75, 1 (2003).

[22] K. Ono, D. G. Austing, Y. Tokura, and S. Tarucha, Science 297, 1313 (2002).

[23] S. R. Schofield et al., Phys. Rev. Lett. 91, 136104 (2003).

[24] J. R. Tucker and T.-C. Shen, Quantum Inf. Comput. 1, 129 (2001).

[25] A. D. Greentree, A. R. Hamilton, and F. Green, Phys. Rev. B 70, 041305(R) (2004).

[26] Y. Aharonov and J. Anandan, Phys. Rev. Lett. 58, 1593 (1987).

[27] J. F. Poyatos and J. I. Cirac, Phys. Rev. Lett. 78, 390 (1997).

[28] M. D. Bowdery et al., Phys. Lett. A 294, 258 (2002).

[29] S. G. Schirmer, A. D. Greentree, V. Ramakrishna, and H. Rabitz, J. Phys. A 35, 8315 (2002).

[30] C. J. Wellard, Private Communication, 2004.

[31] B. Koiller, X. Hu, and S. Das Sarma, Phys. Rev. Lett. 88, 027903 (2002).

[32] C. J. Wellard et al., Phys. Rev. B 68, 195209 (2003).

[33] These figures are for numerical simulations with time step $\Delta t=0.005$ and can be improved further by decreasing $\Delta t$. 
[34] This value seems realistic to allow for a metal barrier sufficiently thick to provide adequate screening and sufficient distance between the quantum dots and the barrier to minimize barrier-induced decoherence.
[35] In practice, the effective Coulomb interaction should be determined experimentally for a given system. 